\title{
High-carbohydrate diet-induced myocardial remodelling in rats
}

\author{
Esma N Okatan MSc, Sule Kizil MSc ${ }^{1}$, Hilal Gokturk MD², Belgin Can MD ${ }^{1}$, Belma Turan PhD
}

\author{
EN Okatan, S Kizil, H Gokturk, B Can, B Turan. High-carbohydrate \\ diet-induced myocardial remodelling in rats. Curr Res Cardiol \\ 2015;2(1):5-10.
}

BACKGROUND: A high-carbohydrate diet leads to the metabolic syndrome (MetS), which leads to an increased risk for cardiovascular dysfunction; however, the effect of high-carbohydrate diets on systemic metabolism has not yet been fully determined. It has been observed that abnormal fatty acid metabolism and increased oxidative stress play a role in the pathogenesis of MetS-related cardiovascular diseases.

OBJECTIVE: To examine the effects of high sucrose intake on left ventricular contractility and structure of heart tissue.

METHODS: MetS was induced in male rats with drinking water supplemented with $32 \%$ sucrose for 16 weeks. Oral glucose tolerance test results and parameters related to insulin resistance were used to validate MetS.

RESULTS: Body weight and blood glucose levels were higher in the MetS group compared with age-matched controls. The increased serum leptin and triglyceride levels and decreased ghrelin levels further supported the existence of MetS in the MetS group. The ratio of total oxidant status to total antioxidant status measured in serum was also higher in the MetS group compared with the control group. The hemodynamic parameters of the MetS group, such as heart rate, and systolic and diastolic blood pressure levels, were markedly higher in the MetS group, while left ventricular developed pressure was significantly diminished with prolonged time course. Moreover, these functional alterations in cardiac preparations were further supported with structural changes such as significant increases in myofibril undulation and increased lipid droplets.

CONCLUSIONS: These data highlight the link between high carbohydrate intake, MetS and cardiac dysfunction, in part due to increased systemic oxidative stress and lipid deposition in the heart tissue.

Key Words: Heart dysfunction; Insulin resistance; Metabolic syndrome; Oxidative stress

Studies have shown that the consumption of sugar-sweetened beverages (SSBs) has been associated with excess body weight and increased risk for MetS or type 2 diabetes (13-15). In a meta-analysis by Malik et al (13), it was reported that daily consumption of SSBs was associated with a $15 \%$ increased risk for development of type 2 diabetes. In addition, Shulze et al (16) found that individuals who consumed $>1$ SSB per day had an $83 \%$ higher risk for diabetes compared with those who consumed $<1$ per month (16). With regard to the cardiovascular effects of high sugar intake, a $20 \%$ increase in coronary artery disease among men and women has been reported $(17,18)$. However, the process by which high sugar intake alters the synchrony of the cardiovascular system is not yet clear. In addition, although a high-carbohydrate diet leads to MetS, with a resulting increased risk for cardiovascular dysfunction, and the effects of high sucrose intake on systemic metabolism have been studied, myocardial capacity for sucrose uptake and its metabolism have not been well characterized to date. Therefore, the aims of the present study are as follows: to evaluate the effects of high sucrose intake in a rat model on systemic metabolic parameters of rats, including blood glucose and insulin levels as well as hormone levels such as ghrelin and leptin levels; and to demonstrate structural and functional abnormalities of the heart in these animal models compared with those of age-matched controls.

\section{METHODS}

Experimental protocols for MetS in the rats

Adult male two-month-old Wistar rats were used. MetS was induced in the rats by providing drinking water containing sucrose (32\%) for a 16-week period, as described previously (19).

All animals were exposed to a $12 \mathrm{~h}$ light/dark cycle and were given free access to tap water (control [CON] group) or tap water with sucrose added (MetS group). They were fed standard chow ad wilar articture and function, particularly left ventiis a goal of cardiovascular disease prevention $(10-12)$.

${ }^{1}$ Departments of Biophysics, Histology-Embriology, Ankara University, Faculty of Medicine; ${ }^{2}$ Department of Histology-Embriology, Yildirim Beyazit University Faculty of Medicine, Ankara, Turkey

Correspondence: Dr Belma Turan, Department of Biophysics, Faculty of Medicine, Ankara University, 06100 Ankara, Turkey. Telephone 90-312-5958186, e-mail belma.turan@medicine.ankara.edu.tr 
libitum daily. All animals were housed in standard rat cages (three per cage). The experimental protocol and handling of animals were approved by the Ankara University ethics committee (No: 2012-5-35 and 2007-11-35).

\section{Serum biochemical parameters}

Rats were fasted overnight and anesthetized using pentobarbital sodium $(50 \mathrm{mg} / \mathrm{kg}$ intraperitoneally). Blood samples were collected after excision of the heart and serum was separated by centrifugation and stored at $-80^{\circ} \mathrm{C}$. Serum triglyceride levels were determined using a colourimetric commercial kit (10010303; Cayman Chemical, USA). Serum insulin levels were assessed using an enzyme immunoassay kit (A05105; SPIBIO, France). IR and $\beta$ cell function were evaluated using the homeostatic model assessment (HOMA) index: HOMA-IR was calculated using the following formula (20):

$$
\text { (insulin }[\mu \mathrm{U} / \mathrm{mL}] \times \text { glucose }[\mathrm{mmol} / \mathrm{L}]) / 22.5
$$

HOMA- $\beta$ was calculated by the following formula (20):

$$
(20 \times \text { insulin }[\mu \mathrm{U} / \mathrm{L}]) /(\text { glucose }[\mathrm{mmol} / \mathrm{L}]-3.5)
$$

\section{Oral glucose tolerance test}

Rats were deprived of food overnight with free access to tap water (with no sucrose added) and received an orogastric gavage of $1 \mathrm{~g} / \mathrm{kg}$ glucose in double-distilled water, as described previously (21). Blood samples were collected from a small cut at the tip of the tail immediately before and at $15 \mathrm{~min}, 30 \mathrm{~min}, 60 \mathrm{~min}$ and $120 \mathrm{~min}$ after glucose administration. Blood glucose levels were assessed using standard glucose test strips (GlucoCheck Analyzer, Roche, Germany) and blood insulin levels were measured using an insulin enzyme immunoassay kit (A05105, SPIBIO, France). The total area under the curve was calculated using GraphPad Prism.

\section{Measurement of arterial pressure with tail-cuff method}

Systolic and diastolic blood pressures were measured indirectly using the tail-cuff method (NIBP200-A; BIOPAC Systems Inc, USA) as previously described (22).

\section{Western blot analysis}

Protein expression levels of serum leptin and ghrelin were determined using Western blot analysis. Equal protein amounts of serum samples were loaded and separated on $15 \%$ sodium dodecyl sulphate polyacrylamide gel electrophoresis gels under reducing conditions. After electrophoresis ( $150 \mathrm{~V}$ for $3 \mathrm{~h}$ at room temperature), samples were electroblotted onto a polyvinylidene fluoride membrane by wet transfer in Towbin buffer ( $200 \mathrm{~mA}$ for $1 \mathrm{~h}$ ). $\beta$-actin levels were quantified as a loading control for primary antibody signals of leptin or ghrelin. Immunoreactive protein bands were visualized using an enhanced chemiluminescence detection system.

Assessment of oxidative stress and antioxidant status in serum samples Total oxidant status (TOS) and total antioxidant status (TAS) in the serum samples were measured using commercial kits (RL0017 and RL0024, respectively; Rel Assay Diagnostics, Turkey) as previously described (23). TOS or TAS assays were calibrated with $\mathrm{H}_{2} \mathrm{O}_{2}$, and the results were expressed in terms of $\mu \mathrm{mol} \mathrm{H}_{2} \mathrm{O}_{2}$ equivalent/L or with Trolox standard (an analogue of vitamin E) and the results were expressed as mmol Trolox equivalent/L, respectively.

\section{Histological examination}

Light microscopic evaluation was performed as described previously (24). Briefly, the excised heart samples were fixed in phosphate buffer $10 \%$ formaldehyde and dehydrated through a series of alcohol solutions. Following clearing and paraffin infiltration processes, the embedded samples were sectioned to $4 \mu \mathrm{m}$ thickness using a Leica (Germany) RM 2125RT microtome. The sections were stained with hematoxylin and eosin and Masson's trichrome histological stains. To stain lipids, heart samples were fixed in $4 \%$ paraformaldehyde for $24 \mathrm{~h}$, and were subsequently incubated with a solution of $1.2 \mathrm{M}$ sucrose and $0.1 \%$ paraformaldehyde at $4^{\circ} \mathrm{C}$ until they sank ( $1 \mathrm{~h}$ to $24 \mathrm{~h}$ ). The samples were embedded in cryomatrix (Sigma, USA), sectioned to $10 \mu \mathrm{m}$ thickness and stained with Oil Red-O stain. All samples were photographed using an AxioCam MRc5 (Carl Zeiss, Germany) digital vision system.

Using light microscopy, the diameter of cardiomyocytes, intracellular vacuolization and lipid deposition, and arrangement of myofibrils were evaluated. To compare cardiomyocyte diameters, one preparation was chosen randomly for every test animal from each group (CON and MetS). Diameters of 10 different muscle fibres were measured (from the two nearest points of one fibre) from five different areas at $\times 40$ magnification; thus, a mean of 50 measurements belonging to each animal were statistically evaluated. Intracellular lipid deposition was evaluated histologically using light microscopy, and scored as none $=0$, weak $=+1$, mild $=+2$, moderate $=+3$ and strong $=+4$.

Examination of left ventricle function using Langendorff perfusion Rats were anesthetized with pentobarbital sodium $(50 \mathrm{mg} / \mathrm{kg})$ then euthanized. The hearts were rapidly excised and perfused according to the Langendorff procedure, as described (25). The perfusion medium, containing $119 \mathrm{mM} \mathrm{NaCl}, 4.8 \mathrm{mM} \mathrm{KCl}, 1.0 \mathrm{mM} \mathrm{CaCl}_{2}, 1.2 \mathrm{mM} \mathrm{MgSO}_{4}$, $1.2 \mathrm{mM} \mathrm{KH}_{2} \mathrm{PO}_{4}, 20 \mathrm{mM} \mathrm{NaHCO}_{3}$, and $10 \mathrm{mM}$ glucose, was gassed with $95 \% \mathrm{O}_{2} / 5 \% \mathrm{CO}_{2}$ and maintained at $\mathrm{pH} 7.4$ at $37^{\circ} \mathrm{C}$. The hearts were paced at 300 beats/min (by a square wave of twice the threshold voltage for $1.5 \mathrm{~ms}$ ) with an electrical stimulator (DCS, Harvard), and the coronary flow rate was maintained at $10 \mathrm{~mL} / \mathrm{min}$. To measure the left ventricular pressure, the left atrium was removed and a latex balloon connected to a pressure transducer (model P23XL; Spectramed, USA) was inserted through the mitral valve into the left ventricle. The balloon was filled with water and adjusted to the left ventricular enddiastolic pressure of $20 \mathrm{mmHg}$ to $30 \mathrm{mmHg}$, depending on the heart. Values of the left ventricular developed pressure (LVDP), rates of pressure development $(+\mathrm{dP} / \mathrm{d} t)$ and rates of pressure decay $(-\mathrm{dP} / \mathrm{d} t)$ were recorded online using a Biopac data acquisition system (Biopac Systems, USA). The time to peak LVDP and its half-relaxation time were also measured from each pressure trace.

\section{Chemicals and statistics}

Chemicals were obtained from Sigma-Aldrich (USA) unless otherwise noted. Leptin and ghrelin antibodies were obtained from Abcam (USA). $\beta$-actin antibody and enhanced chemiluminescence were purchased from Santa Cruz Biotechnology (USA).

The results are expressed as mean \pm SEM. Statistical significance was evaluated using a one-way ANOVA followed by Tukey post-test; $\mathrm{P}<0.05$ was considered to be statistically significant.

\section{Validation of MetS}

\section{RESULTS}

Sixteen weeks following 32\% sucrose supplementation via drinking water in addition to their daily diet, the MetS group exhibited significant weight gain (approximately 20\%), high blood glucose and serum insulin levels $(\mathrm{P}<0.05)$, and higher water consumption, compared with age-matched controls (Table 1). The serum triglyceride level in the MetS group was significantly higher than in the CON group. In addition, heart weight and its ratio to body weight were significantly higher in the MetS group compared with the CON group. Therefore, increased body weight and intra-abdominal fat, which are important characteristics of MetS, were observed as markers of IR and high blood glucose level; there was marked hypertrophy in all animals ingesting high-sucrose drinking water.

To detect impaired glucose tolerance and IR in MetS rats, HOMA-IR and HOMA $\beta$ were measured, and oral glucose tolerance test results were calculated using the area under curve. HOMA-IR and oral glucose tolerance tests, but not HOMA- $\beta$, demonstrated the marked 
TABLE 1

Parameters of the metabolic syndrome in rats supplemented with high sucrose in drinking water (MetS) and control rats (CON)

\begin{tabular}{|c|c|c|}
\hline & \multicolumn{2}{|c|}{ Group } \\
\hline & CON & MetS \\
\hline Body weight $^{\star}, g$ & $328.2 \pm 7.2$ & $357.2 \pm 8.8^{\ddagger}$ \\
\hline Heart weight $^{\dagger}, \mathrm{g}$ & $1.5 \pm 0.1$ & $2.1 \pm 0.1^{\ddagger}$ \\
\hline Heart weight/body weight ${ }^{\dagger}, \times 10^{-3}$ & $4.3 \pm 0.3$ & $5.4 \pm 0.5^{\ddagger}$ \\
\hline Glucose ${ }^{\star}, \mathrm{mmol} / \mathrm{L}$ & $5.05 \pm 0.1$ & $6.77 \pm 0.4^{\ddagger}$ \\
\hline Triglycerides $^{\dagger}, \mathrm{mmol} / \mathrm{L}$ & $0.37 \pm 0.02$ & $0.5 \pm 0.05^{\ddagger}$ \\
\hline Insulin $^{\dagger}, \mathrm{pmol}^{\prime} \mathrm{L}$ & $0.23 \pm 0.05$ & $0.57 \pm 0.05^{\ddagger}$ \\
\hline HOMA-IR ${ }^{\dagger}$ & $9.6 \pm 1.6$ & $27.8 \pm 3.8^{\ddagger}$ \\
\hline HOMA- $\beta^{\dagger}$ & $0.5 \pm 0.1$ & $0.5 \pm 0.1$ \\
\hline OGTT, area under the curve* & $592 \pm 25$ & $868 \pm 37^{\ddagger}$ \\
\hline
\end{tabular}

Data presented as mean \pm SEM. ${ }^{*} \mathrm{CON}, n=30$; MetS, $n=44 ;{ }^{\dagger} \mathrm{CON}, n=10$; MetS, $n=10 ;{ }^{\ddagger} P<0.05$ (unpaired Student's t test) versus CON group. $\beta \beta$-cell function; HOMA Homeostatic model assessment; IR Insulin resistance; OGTT Oral glucose tolerance test

impaired glucose tolerance and IR in the MetS group (Table 1). Blood glucose and insulin levels before and after ( $15 \mathrm{~min}, 30 \mathrm{~min}, 60 \mathrm{~min}$ and $120 \mathrm{~min}$ ) orogastric gavage of glucose $(1 \mathrm{~g} / \mathrm{kg})$ in the MetS group compared with the CON group are presented in Figure 1A and C, respectively. The percentage contribution of the groups in the changes of blood glucose and serum insulin levels at the time points of $15 \mathrm{~min}$ to $30 \mathrm{~min}$, $30 \mathrm{~min}$ to $60 \mathrm{~min}$, and $60 \mathrm{~min}$ to $120 \mathrm{~min}$ are presented in Figure $1 \mathrm{~B}$ and $1 \mathrm{D}$, respectively.

Leptin and ghrelin levels as markers of MetS in rats

It is well accepted that the hormones ghrelin and leptin have a major influence on energy balance. Therefore, the protein expression levels of these two hormones were measured in serum of the experimental rats. As presented in Figure 2A, ghrelin level was decreased and leptin level was increased in the high sucrose-supplemented group, compared with that of the control (both $\mathrm{P}<0.05$ ).

To examine the oxidative status in high sucrose supplementation, serum TOS and TAS were measured in both groups. Significantly increased TOS levels and decreased TAS levels were observed in the MetS group compared with the CON group (Figure 2B).

\section{Morphological findings in the MetS rat heart}

After excision, the left ventricles from both groups were examined under light microscopy. Myocardial histology from MetS rats was markedly different from the controls (Figure 3A). In the crosssectional examinations, marked pale staining and reduced uptake of eosin in some myofibrils as well as marked heterogenity in the cytoplasm were observed in the MetS group (Figure 3A). Myofibril diameters were measured and compared between the groups; diameters in the MetS group were smaller but did not reach significance (Figure 3B). Evaluation of longitudinal sections revealed some differences; in the MetS group, the myofibrils showed some irregularities and undulations, and some appeared to have lost their integrity (Figure 3A).

Following Masson's trichrome staining, some cytoplasmic disorganization was observed in the MetS group, and the connective tissue was increased around myofibrils compared with the CON group (Figure 3C). On examination with Oil-Red-O staining, significant intracellular lipid inclusions, which spread to extensive areas and appeared dark stained, were observed in the MetS group (Figure 3D). Scoring of intracytoplasmic lipid inclusions for the MetS group compared with the CON group are presented in Figure 3E.

Intracellular lipid depositions was evaluated histologically under light microscopy and the scoring parameters are presented in Table 1.

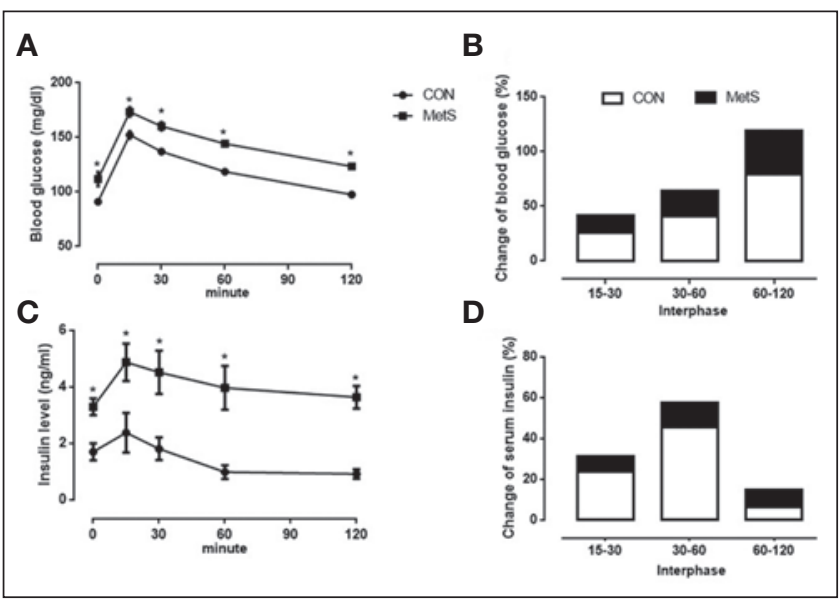

Figure 1) Validation of hyperglycemia and hyperinsulinemia in rats supplemented with high sucrose in drinking water $(\mathrm{MetS})$ and age-matched control rats (CON) with glucose tolerance test. Blood glucose (A) and insulin (C) levels before and after orogastric gavage of $1 \mathrm{~g} / \mathrm{kg}$ glucose at $15 \mathrm{~min}, 30 \mathrm{~min}$, $60 \mathrm{~min}$ and $120 \mathrm{~min}$ following administration in MetS rats compared with CON. Bar graphs representing the percentage contribution of the groups in the changes of blood glucose (B) and serum insulin (D) levels at the time points of 15 to $30 \mathrm{~min}, 30 \mathrm{~min}$ to $60 \mathrm{~min}$, and $60 \mathrm{~min}$ to $120 \mathrm{~min}$, respectively. Data presented as mean ( \pm SEM) values. Number of rats in each group: CON n=30, MetS $n=44$ for glucose measurements ( $\mathrm{A}$ and $\mathrm{B}$ ); $\mathrm{CON} n=10, \mathrm{Met} \mathrm{S} n=10$ for insulin measurements (C and D). *Significant at $\mathrm{P}<0.05$ versus CON group according to unpaired Student's $\mathrm{t}$ tests

A
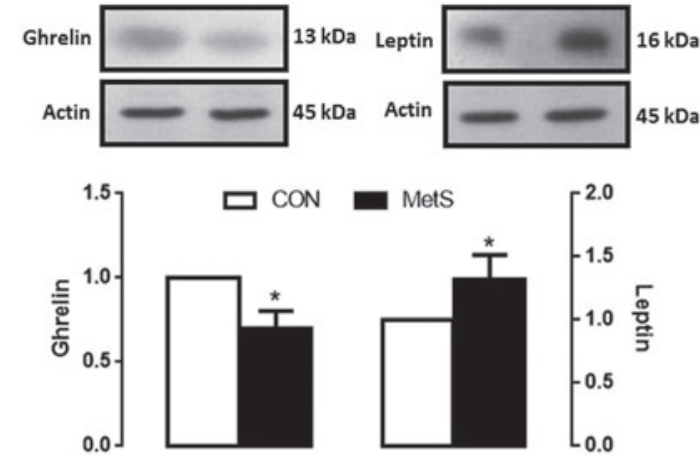

B

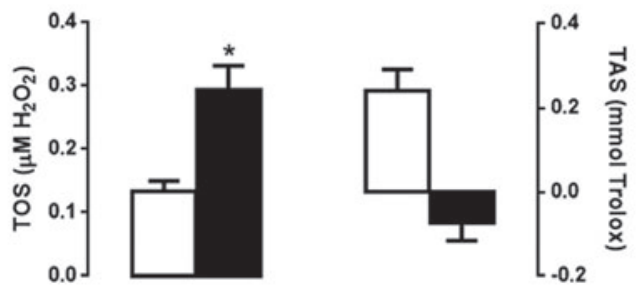

Figure 2) Circulatory oxidative stress and appetite hormone levels in rats supplemented with high sucrose in drinking water (MetS). Serum protein levels of ghrelin (left) and leptin (right) in MetS group rats obtained from Western blot analysis (A). $\beta$-actin for loading control and results expressed as primary antibody signal over the $\beta$-actin signal. (B) Serum total oxidant status (TOS) (left) measured with respect to $\mathrm{H}_{2} \mathrm{O}_{2}$ and the total antioxidant status (TAS) (right) measured with respect to Trolox. Data are presented as mean $( \pm$ SEM). Number of rats in each group: control (CON) $n=6$, MetS $n=6$. *Significant at $P<0.05$ versus $C O N$ group according to unpaired Student's test 


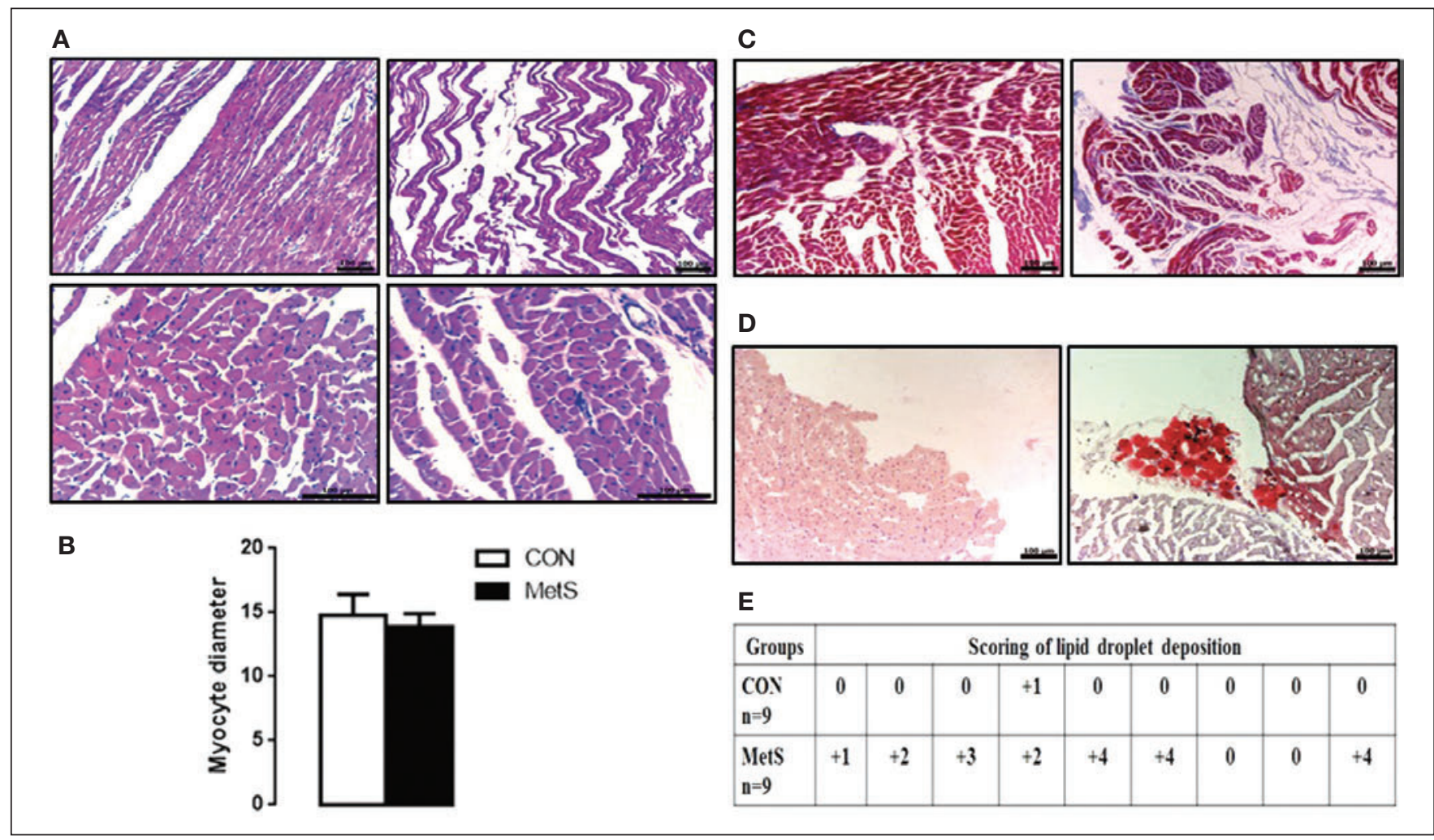

Figure 3) Histological investigation of alterations in the myocardium of rats supplemented with high sucrose in drinking water (MetS) and age-matched control $(\mathrm{CON})$ rats using light microscopy. Micrographs of left ventricular cross section of $\mathrm{CON}$ and $\mathrm{Met} S$ groups using light microscopy. A Light microscopy examination of hematoxylin and eosin-stained heart sections. Myocardial arrangements in longitudinal sections (CON, upper left panel; MetS, upper right panel) reveal undulating myofibrils in MetS. Diameters of the cardiomyocytes evaluated in CON (lower left panel) and MetS (lower right panel) transverse sections (bars = $100 \mu \mathrm{m})$ (B). C Light microscopy examinations represent Masson's trichrome-stained heart sections of CON (left) and MetS (right) groups (bars = 100 um). D Representative Oil-Red-O-stained sections of hearts from CON (left panel) and MetS (right panel) rats. E Scoring table of lipid droplet deposition. Scoring parameters were: none $=0$, weak $=+1$, mild $=+2$, moderate $=+3$ and strong $=+4$. $n=9$ for CON and Met $\mathrm{Sgroups}$

Hemodynamic changes in the MetS rat heart

Systolic and diastolic blood pressures were significantly higher in the MetS group compared with the CON group (Figure 4A). Moreover, the heart rate was markedly higher in the MetS group compared with the CON group (Figure 4B).

Cardiac function was also evaluated using the Langendorff perfusion technique. The basal contractile activity of the heart (LVDP) in MetS rats was found to be significantly depressed compared with the age-matched controls, and the aortic pressure was markedly increased $(\mathrm{P}<0.001)$ (Figure 5A). The time courses of LVDP, such as the time to peak LVDP and its half-relaxation time, were found to be significantly prolonged in the MetS group compared with the CON group (Figure 5B).

In addition, the maximum rate of rise of $\operatorname{LVDP}(+\mathrm{dP} / \mathrm{d} t)$, but not the rate of fall $(-\mathrm{dP} / \mathrm{d} t)$, was significantly decreased in MetS rats compared with the controls (Figure $5 \mathrm{C}$ ). These hemodynamic parameters show important cardiac functional changes, mirroring the structural changes in the MetS group.

\section{DISCUSSION}

Similar to previous research, the present study provides evidence that high sucrose intake profoundly alters cardiac structure and function, in part due to increased systemic oxidative stress in the rats; however, most studies were performed using either high-fat or high-fructose diets (26). Heart rates, as well as systolic and diastolic blood pressures, were significantly higher in MetS rats after 16 weeks of sucrose administration compared with those of the controls. Additionally, LVDP and the aortic pressure in the MetS group were markedly different than the CON group.

Light microscopy examination provided evidence that MetS induced important degenerations in heart tissue. Disorganization of cardiac myofibrils, loss of integrity, decrease in diameter of myofibrils, increase in connective tissue around myofibrils and vessels, intracellular vacuolization, differences in the composition of cell organelles and intracellular lipid inclusion in the MetS group support the targeting of increased oxidative stress not only to entire system but also the heart. The observed changes in the MetS group, such as marked pale staining and reduced uptake of eosin in some myofibrils, and marked heterogenity in the cytoplasm, are linking to intracellular vacuolization and defects of organelles. Some of the findings in the MetS group, such as intracellular vacuolization, have previously been observed in the hearts of rats with streptozotocin-induced diabetes (27). Also, the significantly increased intracellular lipid inclusions in the MetS group indicate increased oxidative stress in the heart tissue, as reported by other authors (28). The observed changes in the hemodynamic parameters highlight important cardiac functional changes, and mirror the structural changes in the MetS group. The changes observed in the heart of MetS rats are consistent with the changes observed in obese individuals (29).

The underlying mechanisms mainly involve the abnormal metabolic state that accompanies diabetes, including IR and hyperglycemia, activating various adverse systems such as oxidative stress, eventually leading to cardiac structural and functional alterations (3-5). By connecting the dynamic relationship between the changes in systemic parameters of the rats and functional parameters of the heart, 


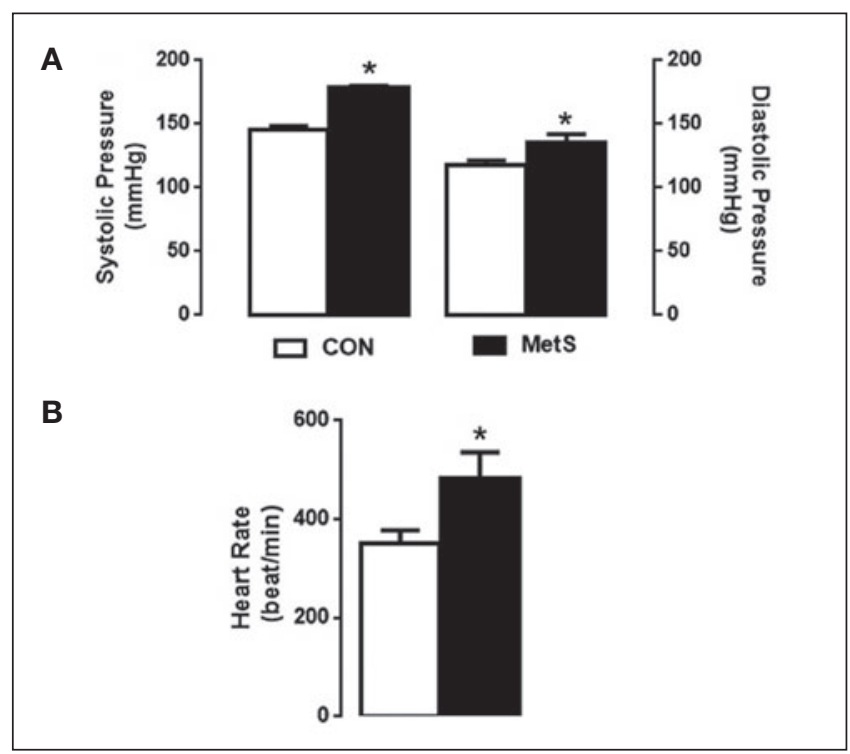

Figure 4) Effect of the metabolic syndrome (MetS) on hemodynamic parameters of the rats. The systolic (left) and diastolic (right) pressure changes (A) and heart rate values (B) in MetS group compared with age-matched controls $(\mathrm{CON})$. Data are presented as mean $( \pm \mathrm{SEM})$. Number of rats in each group: $\mathrm{CON} n=6$, Met $\mathrm{n}=6$. *Significant at $P<0.05$ versus $\mathrm{CON}$, according to unpaired Student's test

we have strong evidence that these alterations can be attributed to increased myocardial reactive oxygen species production and altered $\mathrm{Ca}^{2+}$ handling occurring as a consequence of systemic IR.

Our data have shown that leptin levels were increased while the ghrelin levels were decreased in the MetS group. It is known that leptin is a mediator of long-term regulation of energy balance, suppressing food intake and, thereby, inducing weight loss. On the other hand, ghrelin is a fast-acting hormone that appears to play a role in meal initiation. In obese subjects, the circulating level of the anorexigenic hormone leptin is increased whereas, surprisingly, the level of the orexigenic hormone ghrelin is decreased (30-33). It is now established that some of the obese patients are leptin-resistant $(34,35)$.Our data support those described previously, because the mean body weight of rats in the MetS group was significantly higher compared with those in the CON group. However, the manner in which both the leptin and ghrelin systems contribute to the development or maintenance of obesity is not yet clear, and it may be suggested that possible abnormalities in the leptin and ghrelin systems may contribute to the development of cardiovascular dysfunction via obesity (36).

Data in the literature provide evidence that MetS-associated cardiomyopathy can be distinguished in vivo from diabetic cardiomyopathy by impaired late diastolic function, lower end-diastolic volume, decreased cardiac output and concentric hypertrophy (37). Cardiac function is determined by intrinsic cardiomyocyte function, but also influenced by myoarchitecture, composition and structure of the extracellular matrix, by preload and afterload. It is currently not possible to determine whether the in vivo features of heart dysfunction in MetS are caused by intrinsic cardiomyocyte dysfunction and/or extrinsic factors influencing cardiomyocyte or cardiac contractility. It is noted that our findings are a hallmark of failing myocardium in rats exposed to high sucrose intake. Furthermore, these alterations are in agreement with previous in vivo studies and also with clinical studies (38-40).

Several clinical studies have explored the impact of different numbers of MetS risk factors on the structure and function of the left ventricle (41-43). Using tissue Doppler imaging, Tadic et al (44)

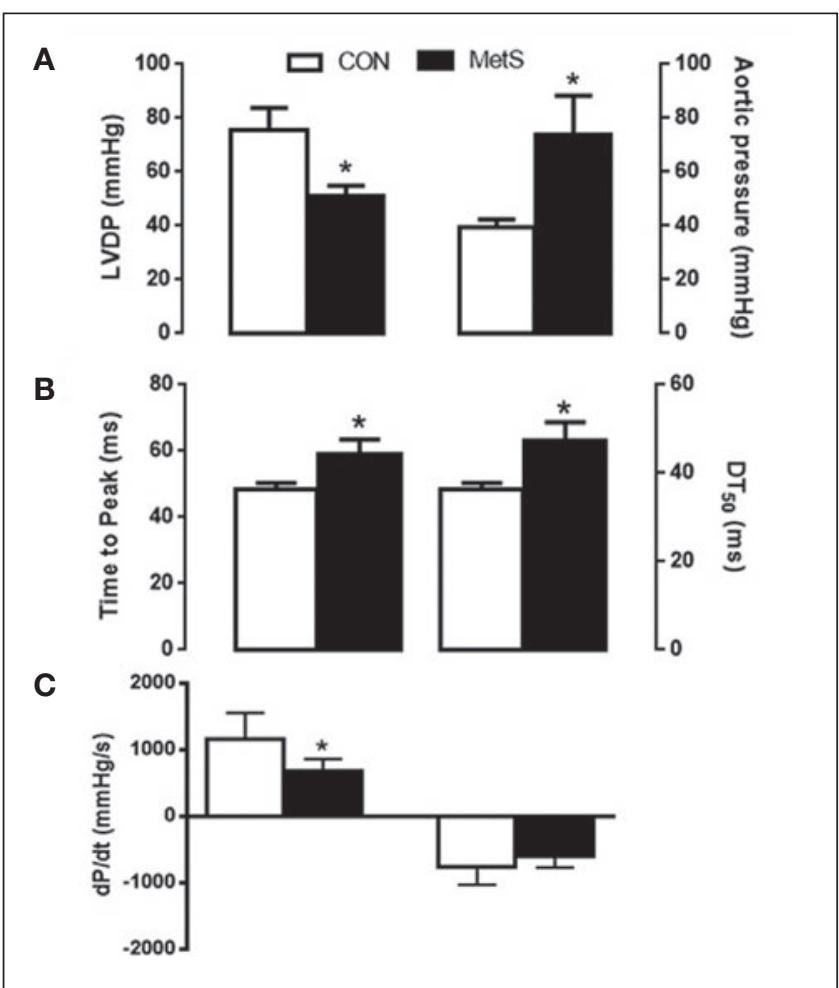

Figure 5) Left ventricular functional parameters and aortic pressure changes in rats supplemented with high sucrose in drinking water (MetS group) compared with age-matched controls (CON group). A Left ventricular developed pressure (LVDP) (left) and aortic pressure (right) changes in MetS rats compared with CON. B Time course of isovolumetric contractions as time to peak (left) and relaxation time at half maximum $\left(D T_{50}\right)$ (right). C Rates of positive $(+d \mathrm{P} / \mathrm{dt})$ and negative changes $(-d \mathrm{P} / \mathrm{dt})$ in developed pressure. Data are presented as mean $( \pm$ SEM). Number of rats in each group: $\mathrm{CON} n=6$, Met $\mathrm{n}=6$. Significant at $* P<0.05$ versus CON, according to unpaired Student's t test

revealed an increasing number of consequences of MetS, such as E/A ratio and Tei index on systolic, diastolic and global left ventricular function. It has also been shown that the contribution of these new parameters of left ventricular diastolic function evidently deteriorated with the increasing number of MetS factors (45). To summarize, both experimental and clinical investigations have shown that the structure of the left ventricle is significantly impaired in patients with MetS, together with impaired left ventricular systolic and diastolic. Thus, it can be concluded that the impaired global left ventricular function is actually the result of impairment of several factors, including increased oxidative stress in MetS individuals. The degree of structural and functional damage increased with the number of risk factors for MetS. Further studies are necessary to confirm the influence of MetS on the left ventricular structure and function.

ACKNOWLEDGEMENTS: The sources of funding had no role in study design, data collection and analysis, decision to publish or preparation of the manuscript.

SOURCE OF FUNDING: This study was supported by TUBITAK SBAG-113S466.

DISCLOSURES: No potential conflicts of interest relevant to this article were reported. 


\section{REFERENCES}

1. Volek JS, Fernandez ML, Feinman RD, Phinney SD. Dietary carbohydrate restriction induces a unique metabolic state positively affecting atherogenic dyslipidemia, fatty acid partitioning, and metabolic syndrome. Prog Lipid Res 2008;47:307-18.

2. Bremer AA, Mietus-Snyder M, Lustig RH. Toward a unifying hypothesis of metabolic syndrome. Pediatrics 2012;129:557-70.

3. Dinh W, Lankisch M, Nickl W, et al. Metabolic syndrome with or without diabetes contributes to left ventricular diastolic dysfunction. Acta Cardiol 2011;66:167-74.

4. Gallagher EJ, Leroith D, Karnieli E. Insulin resistance in obesity as the underlying cause for the metabolic syndrome. Mt Sinai J Med 2010;77:511-23.

5. Eckel RH, Barouch WW, Ershow AG. Report of the National Heart, Lung, and Blood Institute-National Institute of Diabetes and Digestive and Kidney Diseases Working Group on the pathophysiology of obesity-associated cardiovascular disease. Circulation 2002;105:2923-8.

6. Dutta K, Podolin DA, Davidson MB, Davidoff AJ. Cardiomyocyte dysfunction in sucrose-fed rats is associated with insulin resistance. Diabetes 2001;50:1186-92.

7. Futh R, Dinh W, Bansemir L, et al. Newly detected glucose disturbance is associated with a high prevalence of diastolic dysfunction: Double risk for the development of heart failure? Acta Diabetol 2009;46:335-8.

8. Isomaa B, Almgren P, Tuomi T, et al. Cardiovascular morbidity and mortality associated with the metabolic syndrome. Diabetes Care 2001;24:683-9.

9. McNeill AM, Rosamond WD, Girman CJ, et al. The metabolic syndrome and 11-year risk of incident cardiovascular disease in the atherosclerosis risk in communities study. Diabetes Care 2005;28:385-90.

10. Aijaz B, Ammar KA, Lopez-Jimenez F, et al. Abnormal cardiac structure and function in the metabolic syndrome: A populationbased study. Mayo Clin Proc 2008;83:1350-7.

11. de Simone G, Palmieri V, Bella JN, et al. Association of left ventricular hypertrophy with metabolic risk factors: The HyperGEN study. J Hypertens 2002;20:323-31.

12. Mureddu GF, Greco R, Rosato GF, et al. Relation of insulin resistance to left ventricular hypertrophy and diastolic dysfunction in obesity. Int J Obes Relat Metab Disord 1998;22:363-8.

13. Malik VS, Popkin BM, Bray GA, et al. Sugar-sweetened beverages and risk of metabolic syndrome and type 2 diabetes: A meta-analysis. Diabetes Care 2010;33:2477-83.

14. Savoca MR, Evans CD, Wilson ME, Harshfield GA, Ludwig DA. The association of caffeinated beverages with blood pressure in adolescents. Arch Pediatr Adolesc Med 2004;158:473-7.

15. Pollock NK, Bundy V, Kanto W, et al. Greater fructose consumption is associated with cardiometabolic risk markers and visceral adiposity in adolescents. J Nutr 2012;142:251-7.

16. Schulze MB, Manson JE, Ludwig DS, et al. Sugar-sweetened beverages, weight gain, and incidence of type 2 diabetes in young and middle-aged women. JAMA 2004;292:927-34.

17. de Koning L, Malik VS, Kellogg MD, et al. Sweetened beverage consumption, incident coronary heart disease, and biomarkers of risk in men. Circulation 2012;125:1735-41, S1.

18. Fung TT, Malik V, Rexrode KM, et al. Sweetened beverage consumption and risk of coronary heart disease in women. Am J Clin Nutr 2009;89:1037-42.

19. Ruiz-Ramirez A, Chavez-Salgado M, Peneda-Flores JA, et al. High-sucrose diet increases ROS generation, FFA accumulation, UCP2 level, and proton leak in liver mitochondria. Am J Physiol Endocrinol Metab 2011;301:E1198-207.

20. Shpilberg Y, Beaudry JL, D'Souza A, et al. A rodent model of rapidonset diabetes induced by glucocorticoids and high-fat feeding. Dis Model Mech 2012;5:671-80.

21. Steen MS, Foianini KR, Youngblood EB, et al. Interactions of exercise training and ACE inhibition on insulin action in obese Zucker rats. J Appl Physiol 1999;86:2044-51.

22. Krege JH, Hodgin JB, Hagaman JR, Smithies O. A noninvasive computerized tail-cuff system for measuring blood pressure in mice. Hypertension 1995;25:1111-5.

23. Ozcinar E, Okatan EN, Tuncay E, Eryilmaz S, Turan B. Improvement of functional recovery of donor heart following cold static storage with doxycycline cardioplegia. Cardiovasc Toxicol 2014;14:64-73.

24. Gokturk H, Ulusu NN, Gok M, et al. Long-term treatment with a beta-blocker timolol attenuates renal-damage in diabetic rats via enhancing kidney antioxidant-defense system. Mol Cell Biochem 2014;395:177-86.

25. Yaras N, Tuncay E, Purali N, et al. Sex-related effects on diabetesinduced alterations in calcium release in the rat heart. Am J Physiol Heart Circ Physiol 2007;293:H3584-92.

26. Mellor KM, Wendt IR, Ritchie RH, Delbridge LM. Fructose diet treatment in mice induces fundamental disturbance of cardiomyocyte $\mathrm{Ca} 2+$ handling and myofilament responsiveness. Am J Physiol Heart Circ Physiol 2012;302: H964-72.

27. Ayaz M, Can B, Ozdemir S, Turan B. Protective effect of selenium treatment on diabetes-induced myocardial structural alterations. Biol Trace Elem Res 2002;89:215-26.

28. Marfella R, Di Filippo C, Portoghese M, et al. Myocardial lipid accumulation in patients with pressure-overloaded heart and metabolic syndrome. J Lipid Res 2009;50:2314-23.

29. Kishi S, Armstrong AC, Gidding SS, et al. Association of obesity in early adulthood and middle age with incipient left ventricular dysfunction and structural remodeling: The CARDIA study (Coronary Artery Risk Development in Young Adults).

JACC Heart Fail 2014;2:500-8.

30. Faraj M, Havel PJ, Phelis S, et al. Plasma acylation-stimulating protein, adiponectin, leptin, and ghrelin before and after weight loss induced by gastric bypass surgery in morbidly obese subjects. J Clin Endocrinol Metab 2003;88:1594-602.

31. McLaughlin T, Abbasi F, Lamendola C, Frayo RS, Cummings DE. Plasma ghrelin concentrations are decreased in insulin-resistant obese adults relative to equally obese insulin-sensitive controls. J Clin Endocrinol Metab 2004;89:1630-5.

32. Hansen TK, Dall R, Hosoda H, et al. Weight loss increases circulating levels of ghrelin in human obesity. Clin Endocrinol (Oxf) 2002;56:203-6.

33. Tschop M, Weyer C, Tataranni PA, et al. Circulating ghrelin levels are decreased in human obesity. Diabetes 2001;50:707-9.

34. Kimber W, Peelman F, Prieur X, et al. Functional characterization of naturally occurring pathogenic mutations in the human leptin receptor. Endocrinology 2008;149:6043-52.

35. Roujeau C, Jockers R, Dam J. New pharmacological perspectives for the leptin receptor in the treatment of obesity. Front Endocrinol (Lausanne) 2014;5:167.

36. Tritos NA, Kissinger KV, Manning WJ, Danias PG. Association between ghrelin and cardiovascular indexes in healthy obese and lean men. Clin Endocrinol (Oxf) 2004;60:60-6.

37. Van den Bergh A, Vanderper A, Vangheluwe P, et al. Dyslipidaemia in type II diabetic mice does not aggravate contractile impairment but increases ventricular stiffness. Cardiovasc Res 2008;77:371-9.

38. Nevelsteen I, Bito V, Van der Mieren G, et al. ACE-inhibition, but not weight reduction restores cardiomyocyte response to beta-adrenergic stimulation in the metabolic syndrome. BMC Cardiovasc Disord 2013;13:51.

39. Van den Bergh A, Vangheluwe P, Vanderper A, et al. Food-restriction in obese dyslipidaemic diabetic mice partially restores basal contractility but not contractile reserve. Eur J Heart Fail 2009;11:1118-25.

40. Alpert MA, Terry BE, Mulekar M, et al. Cardiac morphology and left ventricular function in normotensive morbidly obese patients with and without congestive heart failure, and effect of weight loss. Am J Cardiol 1997;80:736-40.

41. de las Fuentes L, Brown AL, Mathews SJ, et al. Metabolic syndrome is associated with abnormal left ventricular diastolic function independent of left ventricular mass. Eur Heart J 2007;28:553-9.

42. Azevedo A, Bettencourt P, Almeida PB, et al. Increasing number of components of the metabolic syndrome and cardiac structural and functional abnormalities--cross-sectional study of the general population. BMC Cardiovasc Disord 2007;7:17.

43. Janiszewski M, Cudnoch-Jedrzejewska A, Sadkowska K, et al. [Myocardial structure and function in patients with metabolic syndrome - echocardiographic and ergospirometric assessment]. Pol Merkur Lekarski 2008;25:15-8.

44. Tadic M, Ivanovic B, Kostic N, et al. Metabolic syndrome and left ventricular function: Is the number of criteria actually important? Med Sci Monit 2012;18: CR282-9.

45. Masugata H, Senda S, Goda F, et al. Left ventricular diastolic dysfunction as assessed by echocardiography in metabolic syndrome. Hypertens Res 2006;29:897-903. 